\title{
Design and Realization of Oil Rapid Detector
}

\author{
Xin LI ${ }^{1, a}$, Letian Teng ${ }^{2, b}$, Wennan Miao ${ }^{1, c}$ \\ ${ }^{1}$ Guangzhou College of South China University of \\ Technology, Guangzhou, 510800, China;
}

\begin{abstract}
With the enhancement of public awareness of food safety, the rapid detection of edible oil and other oil is in great need today. Using the relative dielectric constant difference among various oils and considering the effect of temperature on oil property, an instrument for rapidly detecting and identifying hogwash oil and edible oil has been developed with the integrative sensor of capacitor and temperature and the minimum system of microcomputer. The instrument which can be used by general public, inspection agency and research institute has the advantages of low-power, high reliability, portable and intuitive demonstration.
\end{abstract}

Keywords-microcomputer; oil; relative dielectric constant; sensor

\section{INTRODUCTION}

Hogwash oil, refers to the life of all kinds of inferior oil. Hogwash oil can be divided into three categories: one is the narrow hogwash oil, will drain in a greasy floater or hotel, restaurant leftovers, leftovers (commonly known as swill) through simple processing, refined oil; two is the inferior pork, pig offal, pigskin etc processing and refining production after the oil; three is used for frying use number exceeds a certain number of food oil, and then be reused or add some new oil after the re used oil into the. The harm of hogwash oil on the human body is great, but the market has differential hogwash oil method, such as by cooling to 0 $5^{\circ} \mathrm{C}$ to observe whether the oil solidification or by garlic fried discrimination, these methods are not accurate or no scientific basis ${ }^{[1]}$. Therefore, to develop quickly and accurately identify hogwash oil and edible oil instrument for food security, has the vital significance to protect the health of people.

The urgent demand for rapid and accurate identification of hogwash oil, this paper based on considering the influence of temperature and liquid level of the oil characteristics, carried out the research of detecting the relative dielectric constant based on the difference of hogwash oil. Research and design a portable oil detector based on temperature and capacitance sensor and MCU minimum system integration, the establishment of form and storage of data through the sensor level control in different temperature and oil characteristics, to achieve a fast and accurate identification of different oils.

\section{THE OVERALL SYSTEM FUNCTION}

\section{A. Design idea}

Because of the relative dielectric constant of the oil is

\author{
${ }^{2}$ Guangdong Province 75260 troops, Shaoguan,512100, \\ China; \\ alixin-820701@163.com, ${ }^{\mathrm{b}} 22923963 @ q q . c o m$, \\ c670324959@qq.com
}

different, different types of oil filled to a bipolar plate capacitor when the capacitance of the capacitor values will be different. Using the advantages of NE555RC oscillation circuit $^{[2]}$ fast, the difference of relative dielectric constant change to the output signal frequency conversion. The frequency is calculated by the system, then get count, according to the numerical size partitioning oil count meter range, and the data is stored in the database of internal microcontroller and PC machine, easy to query, so as to realize the discriminating oil.

\section{B. The system function structure}

The system is composed by upper machine and lower machine. Inside, the lower computer is composed by single chip processor, LCD, language module, the key control module, power supply module, oil sensor module, wireless module; the upper machine is composed by PC database and the LABVIEW interface.

The oil sensor measures the temperature and oscillation signal, then wireless transfer to the lower machine data calculation, analysis and comparison, to identify oil varieties. but also wireless transfer the temperature and oscillation signal measured by the oil sensor to the host computer PC system. Describe data changes by the curve mode LABVIEW software on the host computer, the stability of visual data and also provides a reliable experimental data and compared database to identify oil varieties. The system structure diagram is shown in figure $1:$

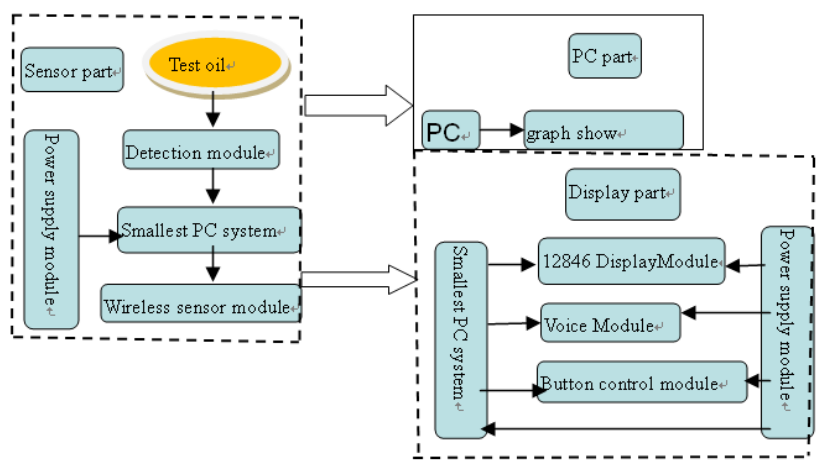

Figure 1. The system structure diagram

\section{THE FUNCTION REALIZATION OF MAIN MODULE}

\section{A. Design of sensor module}

In order to reduce the effect of distribution parameters on the output signal of the sensor, designed for stick type integrated sensor ${ }^{[3]}$. Capacitance sensor is composed of a 
double metal tin copper for capacitor two plates, the two plates are insulated with a thickness of $1 \mathrm{~mm}$ isolation pads, filled of tested oil; capacitance sensor side is pasted on a temperature sensor for the real-time measurement of oil temperature. Has the following advantages of paste type design, as long as the double metal tin copper completely soaked in oil, no matter how placed sensors, metal to metal contact between sensors measured the relative area remains the same, which is to ensure the consistent level. In measurement, as long as the guarantee of capacitance sensor completely in the measured oil, to ensure each measurement between two polar plates of oil equivalent; oil sensor outputs the digital oscillation signal to reflect the dielectric constant and temperature. Schematic diagram of the model is shown in figure 2 :
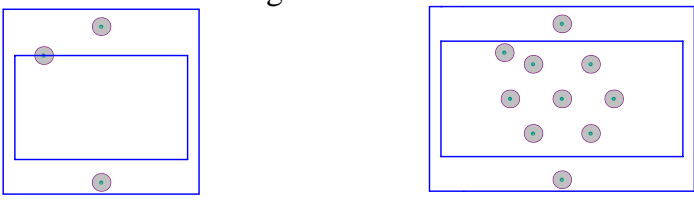

Figure 2. Schematic diagram of sensor model

After the MCU of the sensor part starts, first run the program initialization, then timer, counter start timing and on the rising edge counting, measure temperature at the timing time, then sends signals to the MCU of the receiving part, counter reset, start timing and counting again. The software flow chart is shown in figure 3 :

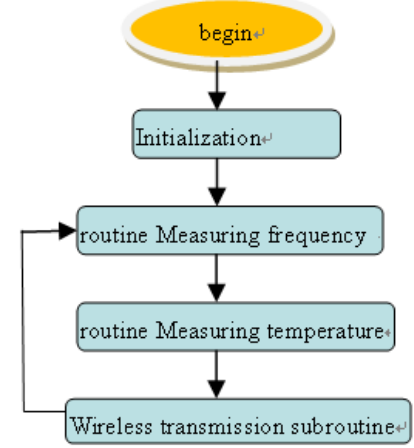

Figure 3. Design flow chart of sensor module program

\section{B. Design frequency calculation module of minimum system}

Using the STC89C52 minimum system ${ }^{[4]}$, the concrete realization method of frequency calculation is: the counting timer of single-chip start counting; when the value capture oscillation signal first pulse timepiece is coming, counter meter $\mathrm{C} 1$, when the timer running to the set time, stop counting, then the counter reset; send signals to the receiving module, and restart when sending completed, counting and timing again.

Using MCU timer and counter function, set the timer 4000*100us, counter measure oscillation signal rising edge in this period of time, stop counting in the end time. Captured for the first time when the measured oscillation signal rising edge, counter record 0 , when capturing oscillation signal rising edge in the end time, record the C1 value. The software design flow chart is shown in figure 4:

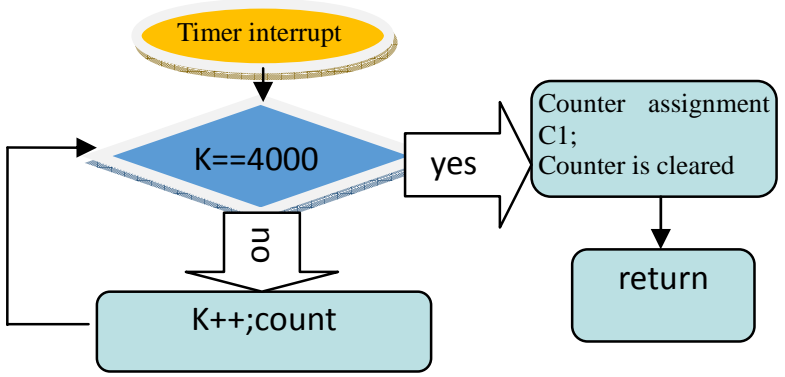

Figure 4. Software design flow chart of frequency calculation module

\section{EXPERIMENTS AND RESULTS ANALYSIS}

\section{A. Data acquisition and analysis processing}

Observe several different oil count value, These three kinds of oil (edible oil, hogwash oil, Engine oil) in the $20 \sim 36^{\circ} \mathrm{C}$ temperature range, have none repeat count value ${ }^{[5]}$, can distinguish between different kinds of oil. Collect the minimum and maximum count values, between three kinds of oil in the $20 \sim 36^{\circ} \mathrm{C}$ temperature range. After analyzing and processing the sample count value, we get the following table 1 :

TABLE 1 SAMPLE DATA

\begin{tabular}{|c|c|c|c|c|c|c|}
\hline counter oil & \multicolumn{3}{|c|}{ edible oil } & \multicolumn{2}{c|}{ hogwash oil } & \multicolumn{2}{c|}{ engine oil } \\
\hline $20-36^{\circ} \mathrm{C}$ & 46598 & 43492 & 49703 & 47212 & 57152 & 51569 \\
\hline
\end{tabular}

B. Test data and results analysis

The results of test data is shown in table 2:

TABLE 2 TEST RESULTS

\begin{tabular}{|c|c|c|c|c|c|c|}
\hline & \multicolumn{2}{|c|}{ hogwash oil } & \multicolumn{2}{|c|}{ edible oil } & \multicolumn{2}{|c|}{ engine oil } \\
\hline No. & $\begin{array}{c}\text { temperatu } \\
\text { re }\end{array}$ & $\begin{array}{c}\text { count } \\
\text { er }\end{array}$ & $\begin{array}{l}\text { temperatu } \\
\text { re }\end{array}$ & $\begin{array}{c}\text { count } \\
\text { er }\end{array}$ & $\begin{array}{c}\text { temperatu } \\
\text { re }\end{array}$ & $\begin{array}{c}\text { count } \\
\text { er }\end{array}$ \\
\hline 1 & 26.0 & 47443 & 26.1 & 46117 & 25.8 & 54252 \\
\hline 2 & 26.0 & 47463 & 26.0 & 46118 & 25.8 & 54212 \\
\hline 3 & 26.0 & 47456 & 26.0 & 46116 & 25.8 & 54288 \\
\hline 4 & 26.0 & 47457 & 26.0 & 46117 & 25.8 & 54190 \\
\hline 5 & 26.0 & 47464 & 26.0 & 46115 & 25.8 & 54189 \\
\hline 6 & 26.0 & 47467 & 26.0 & 46114 & 25.8 & 54173 \\
\hline 7 & 25.9 & 47458 & 26.0 & 46115 & 25.8 & 54158 \\
\hline 8 & 25.9 & 47456 & 26.0 & 46113 & 25.9 & 54239 \\
\hline 9 & 25.9 & 47468 & 26.0 & 46112 & 25.8 & 54142 \\
\hline 10 & 25.9 & 47466 & 26.0 & 46115 & 25.8 & 54173 \\
\hline $\begin{array}{c}\text { Number } \\
\text { of } \\
\text { success } \\
\text { es }\end{array}$ & \multicolumn{2}{|c|}{10} & \multicolumn{2}{|c|}{10} & \multicolumn{2}{|c|}{10} \\
\hline
\end{tabular}

The test results show that, fast lookup table through the method of maximum and minimum temperature range, the instrument can effectively distinguish different oils, fast and reliable detection results. 


\section{CONCLUSION}

This design has finished the setting function, including fast distinguishing oil, voice broadcast detection results, PC curve display. The biggest advantage of the system is using the oil relative dielectric constant difference, and considering the temperature is an important factor to affect the oil characteristics, optimization design of sensor, realized the hogwash oil and edible oil and other oil rapidly differentiate. Oil quick detection instrument of low cost and low power consumption, easy to carry, simple to use, provides a simple and rapid method to detect for ordinary consumers, provides confidence guarantee for food safety of consumers, at the same time can provide detection method for oil food company, fast oil testing production line is qualified, improve production efficiency. Therefore, the significant social benefits.

\section{REFERENCES}

[1] Chen $\mathrm{Li}$, the hogwash oil detection technology research. Technology \& development of chemical industry, Vol. 41 (2012), p. 46. In chinese.

[2] Li Xiangchu. Circuit basis and application of digital (Second Edition), Mechanical Industry Press(2012). In chinese.

[3] Runjing Zhou, sensor and detection technology (Second Edition),Publishing House of electronics industry(2014). In chinese.

[4] Zhiqiang Rao, Caixia Han. Principle and application of single chip microcomputer, Huazhong University of Science and Technology press(2013). In chinese.

[5] Yiguang Li. Trench oil and qualified edible oil differential detection method, Central South University(2013). In chinese. 